momentum transfer. More specifically, we can say that the ratio of the magnetic to the electric radius of the deuteron is

$$
r_{m} / r_{e}=0.93 \pm 0.038
$$

In conclusion we can say that, both from the static magnetic moment and from our measurements, there is some evidence that other contributions besides the impulse approximation have to be taken into account to understand the magnetic structure of the deuteron. We also have some evidence that the form factor connected with this anomalous contribution decreases less rapidly, as the momentum transfer increases, than the form factor obtained from the impulse approximation.

It is a pleasure to thank Professor W. C. Barber for his continuous support and many useful discussions. Our understanding of the problems connected with the deuteron form factors has greatly improved through discussion with various people, in particular, Professor S. Drell, Professor L. I. Schiff, Mr. R. Adler, and Mr. E. Erickson. Dr. G. Vanpraet and Mr. G. Gosta have been of great help in the data taking period. Mr. W. Ewings is responsible for the manufacturing of the particular hydrogen cell that made this experiment possible.

${ }^{*}$ Work supported in part by the joint program of the U. S. Office of Naval Research, the U. S. Atomic Energy Commission, and the U. S. Air Force Office of Scientific Research.
${ }^{1}$ G. A. Peterson and W. C. Barber, Phys. Rev。 $\underline{128}$, 812 (1962).

${ }^{2}$ N. Meister and D. R. Yennie, Phys. Rev. $\underline{130}$, 1210 (1963).

${ }^{3}$ R. Herman and R. Hofstadter, High-Energy Electron Scattering Tables (Stanford University Press, Stanford, California, 1960), see formulas 37 and 48 calculated for $\theta=180^{\circ}$.

${ }^{4}$ E. C. Levinthal, Phys. Rev. 78, 204 (1950).

${ }^{5}$ H. F. Jones, Nuovo Cimento $\underline{27}, 1039$ (1963). For an earlier discussion of the relativistic corrections to the deuteron magnetic moment see also G. Breit and R. M. Thaler, Phys. Rev. 89, 1177 (1953).

${ }^{6}$ M. L. Rustgi, W. Zernik, G. Breit, and D. L. Andrews, Phys. Rev。120, 1881 (1960); M. Matsumoto, Phys. Rev. 129, 1334 (1963); F. Partovi, thesis, Massachusetts Institute of Technology (unpublished).

${ }^{7}$ F. T. Hadjioannou, Phys. Rev. 125, 1414 (1962); J. I. Friedman and H. W. Kendall, Phys. Rev. 129, 2802 (1963); E. F. Erickson and C. Schaerf, Phys. Rev. Letters 11, 432 (1963); see also J. J. de Swart and R. E. Marshak, Phys. Rev. 111, 272 (1958).

${ }^{8} \mathrm{M}$. Gourdin, Nuovo Cimento 28, 533 (1963) (see formulas $8,9,12,13$, and 22). After submittal of this Letter we have been kindly informed by Professor Gourdin that there is a small mistake in his calculations. The corrected formulas give a theoretical value for the deuteron magnetic form factor that is slightly higher than what is indicated in our figures. However, the correction is not appreciable in the momentum range of interest to us and does not alter at all the conclusions of this paper.

${ }^{9}$ We are grateful to Professor G. Breit for having supplied us the results of their calculations prior to publication.

${ }^{10}$ D. J. Drickey and L. N. Hand, Phys. Rev. Letters $\underline{9}, 521$ (1962).

\title{
SOLAR NEUTRINOS. I. THEORETICAL*
}

\author{
John N. Bahcall \\ California Institute of Technology, Pasadena, California
}

(Received 6 January 1964)

The principal energy source for main-sequence stars like the sun is believed to be the fusion, in the deep interior of the star, of four protons to form an alpha particle. ${ }^{1}$ The fusion reactions are thought to be initiated by the sequence ${ }^{1} \mathrm{H}(p$, $\left.e^{+} \nu\right)^{2} \mathrm{H}(p, \gamma)^{3} \mathrm{He}$ and terminated by the following sequences: (i) ${ }^{3} \mathrm{He}\left({ }^{3} \mathrm{He}, 2 p\right)^{4} \mathrm{He}$; (ii) ${ }^{3} \mathrm{He}(\alpha, \gamma){ }^{7} \mathrm{Be}-$ $\left(e^{-} \nu\right)^{7} \mathrm{Li}(p, \alpha)^{4} \mathrm{He}$; and (iii) ${ }^{3} \mathrm{He}(\alpha, \gamma)^{7} \mathrm{Be}(p, \gamma)^{8} \mathrm{~B}-$ $\left(e^{+} \nu\right)^{8} \mathrm{Be}^{*}(\alpha)^{4} \mathrm{He}$. No direct evidence for the existence of nuclear reactions in the interiors of stars has yet been obtained because the mean free path for photons emitted in the center of a star is typically less than $10^{-10}$ of the radius of the star. Only neutrinos, with their extremely small interaction cross sections, can enable us to see into the interior of a star and thus verify directly the hypothesis of nuclear energy generation in stars.

The most promising method ${ }^{2}$ for detecting solar neutrinos is based upon the endothermic reaction $(Q=-0.81 \mathrm{MeV}){ }^{37} \mathrm{Cl}\left(\nu_{\text {solar }}, e^{-}\right)^{37} \mathrm{Ar}$, which was first discussed as a possible means of detecting neutrinos by Pontecorvo ${ }^{3}$ and Alvarez. ${ }^{4}$ In this note, we predict the number of absorptions of 
solar neutrinos per terrestrial ${ }^{37} \mathrm{Cl}$ atom by combining results of recent theoretical investigations $^{5-7}$ of the solar neutrino fluxes with calculations ${ }^{8}$ of the relevant neutrino absorption cross sections on ${ }^{37} \mathrm{Cl}$. The result of a preliminary experiment by Davis ${ }^{2}$ is then used to set an upper limit on the central temperature of the sun and also to give information about the structure of ${ }^{4} \mathrm{Li}$ and its role in the proton-proton chain.

The neutrino fluxes from the hydrogen-burning reactions described in the first paragraph have recently been calculated using detailed models of the sun ${ }^{5,6}$ and the effects of uncertainties in nuclear cross sections, as well as solar composition, opacity, and age, have been determined by Sears. ${ }^{7}$ The most important predictions are these (uncertainties estimated from the work of Sears $\left.{ }^{7}\right): \varphi_{\nu}\left({ }^{7} \mathrm{Be}\right)=(1.2 \pm 0.5) \times 10^{+10}$ neutrinos per $\mathrm{cm}^{2}$ per sec and $\varphi_{\nu}\left({ }^{8} \mathrm{~B}\right)=(2.5 \pm 1) \times 10^{+7}$ neutrinos per $\mathrm{cm}^{2}$ per sec, at the earth's surface.

The cross sections for ${ }^{7} \mathrm{Be}$ and ${ }^{8} \mathrm{~B}$ neutrinos to produce transitions from the ground state of ${ }^{37} \mathrm{Cl}$ to the ground state of ${ }^{37} \mathrm{Ar}$ can readily be calculated from known quantities; the results are ${ }^{8}$ $\sigma_{g}\left({ }^{7} \mathrm{Be}\right)=1.5 \sigma_{0}$ and $\bar{\sigma}_{g}\left({ }^{8} \mathrm{~B}\right)=3.9 \times 10^{+2} \sigma_{0}$, where $\sigma_{0}=1.91 \times 10^{-46} \mathrm{~cm}^{2}$ is a convenient combination of ground-state parameters and $\bar{\sigma}\left({ }^{8} \mathrm{~B}\right)$ has been averaged over the ${ }^{8} \mathrm{~B}$ neutrino spectrum. Three excited states ${ }^{8}$ in ${ }^{37} \mathrm{Ar}$ also have large matrix elements for neutrino absorption by the ground state of ${ }^{37} \mathrm{Cl}$ (which is a $d_{3 / 2}{ }^{3}, J=3 / 2^{+}, T=3 / 2$ state); the three excited states of importance in ${ }^{37} \mathrm{Ar}$ are (with their expected energies) (i) $J=1 / 2^{+}$, $T=1 / 2(1.4 \mathrm{MeV})$; (ii) $J=5 / 2^{+}, T=1 / 2(1.6 \mathrm{MeV})$; and (iii) $J=3 / 2^{+}, T=3 / 2(5.1 \mathrm{MeV})$. The $J=3 / 2^{+}$, $T=3 / 2$ excited state of ${ }^{37} \mathrm{Ar}$ is the analog state of the ground state of ${ }^{37} \mathrm{Cl}$; hence the transition from the ground state of ${ }^{37} \mathrm{Cl}$ to the $5.1-\mathrm{MeV}$ excited state of ${ }^{37} \mathrm{Ar}$ is superallowed and has a large matrix element for neutrino absorption. The calculated absorption cross sections ${ }^{8}$ averaged over the ${ }^{8} \mathrm{~B}$ neutrino spectrum ${ }^{10}$ are, in order of increasing excitation energy, $\bar{\sigma}\left({ }^{8} \mathrm{~B}\right) / \sigma_{0}=0.96 \times 10^{+3}$, $1.3 \times 10^{+3}$, and $4.4 \times 10^{+3}$. The net uncertainty in the magnitude of the sum of the above cross sections is estimated to be about $25 \% \cdot{ }^{8,11}$

The total predicted number of absorptions per terrestrial ${ }^{37} \mathrm{Cl}$ atom per second, using the above estimates for fluxes and cross sections, is found to be

$$
\sum \varphi_{\nu}(\operatorname{solar})_{\sigma_{\text {abs }}}=(4 \pm 2) \times 10^{-35} \mathrm{sec}^{-1}
$$

Only about $10 \%$ of the predicted number of absorp- tions is due to ${ }^{7} \mathrm{Be}$ neutrinos, although the ${ }^{7} \mathrm{Be}$ neutrino flux is predicted to be approximately 500 times the ${ }^{8} \mathrm{~B}$ neutrino flux. ${ }^{12}$ The solar value of $\sum \varphi \bar{\sigma}$ given by Eq. (1) is at least several orders of magnitude greater than one would expect from cosmic neutrinos ${ }^{13}$ or from neutrinos produced in the earth's atmosphere by the decay of cosmic ray secondaries. ${ }^{13,14}$

The ${ }^{8} \mathrm{~B}$ neutrino flux is extremely sensitive $\mathrm{5}^{5,12}$ to the central temperature of the sun because of the large Coulomb barrier, compared to solar thermal energies, for the reaction ${ }^{7} \mathrm{Be}(p, \gamma)^{8} \mathrm{~B}$ of sequence (iii). An upper limit on the central temperature of the sun can therefore be derived by combining the experimental upper limit already obtained by Davis, ${ }^{2}$ on the number of solar neutrinos captured per terrestrial ${ }^{37} \mathrm{Cl}$ atom, with $\mathrm{Eq}$. (1) and the known temperature dependence of the ${ }^{7} \mathrm{Be}(p, \gamma)^{8} \mathrm{~B}$ reaction. In this way we find that the central temperature of the sun is less than 20 million degrees ${ }^{5}$ and that a measurement of the ${ }^{8} \mathrm{~B}$ neutrino flux accurate to $\pm 50 \%$ would determine the central temperature to better than $\pm 10 \%$.

The role of ${ }^{4} \mathrm{Li}$ in the proton-proton chain has long been recognized as an important astrophysical problem, ${ }^{1,15}$ but one that has not yet been solved by direct nuclear physics experiments. The upper limit obtained by Davis ${ }^{2}$ on the number of solar neutrinos captured per terrestrial ${ }^{37} \mathrm{Cl}$ atom can be used, however, to show that ${ }^{4} \mathrm{Li}$ does not play a significant role in the proton-proton chain in the sun. The relevant cross section for neutrino absorption (with $q_{\nu} \max ^{20} \mathrm{MeV}$ ) is ${ }^{8}$ $\bar{\sigma}\left({ }^{4} \mathrm{Li}\right)=2 \times 10^{-42} \mathrm{~cm}^{2}$ and hence $\varphi_{\nu}\left({ }^{4} \mathrm{Li}\right) \leqslant 2 \times 10^{+8}$ neutrinos per $\mathrm{cm}^{2}$ per sec. The fraction of terminations of the proton-proton chain that occur via ${ }^{4} \mathrm{Li}$ can be calculated ${ }^{16}$ as a function of the energy, $E_{r}$, by which the mass of the ground state of ${ }^{4} \mathrm{Li}$ exceeds the mass of ${ }^{3} \mathrm{He}$ plus a proton. One can also calculate an upper limit on the fraction of terminations that occur via ${ }^{3} \mathrm{He}(p$, $\gamma)^{4} \mathrm{Li}\left(\beta^{+} \nu\right)^{4} \mathrm{He}$ by comparing the above upper limit on $\varphi_{\nu}\left({ }^{4} \mathrm{Li}\right)$ (multiplied by $17 \mathrm{MeV}$, the thermal energy release in such a termination) with the observed solar constant $\left(8.7 \times 10^{+11} \mathrm{MeV} \mathrm{cm}^{-2}\right.$ $\mathrm{sec}^{-1}$ ). In this way we find that $E_{r} \geqslant 20 \mathrm{keV}^{17}$ and conclude that ${ }^{4} \mathrm{Li}$ participates in at most $0.2 \%$ of the proton-proton terminations in the sun.

The author is grateful to the entire staff of Kellogg Radiation Laboratory for enthusiastic support and stimulating comments. It is a pleasure to acknowledge many valuable suggestions 
by R. Davis, Jr.

${ }^{*}$ Work supported in part by the U. S. Office of Naval Research and in part by the National Aeronautics and Space Administration.

${ }^{1}$ See, for example, W. A. Fowler, Mem. Soc. Roy. Sci. Liege $3,207(1960)$. The CNO cycle is responsible for only a few percent of the energy generation in the sun and the relatively low-energy neutrinos produced by this cycle are unimportant for solar neutrino detection with ${ }^{37} \mathrm{Cl}$.

${ }^{2} \mathrm{R}$. Davis, Jr., following Letter [Phys. Rev. Letters $12,303(1964)]$. See also R. Davis, Jr., Phys. Rev. 97,766 (1955).

${ }^{3}$ B. Pontecorvo, National Research Council of Canada Report No. P.D. 205, 1946 (unpublished), reissued by the U. S. Atomic Energy Commission as document 200-18787.

${ }^{4}$ L. W. Alvarez, University of California Radiation Laboratory Report No. UCRL-328, 1949 (unpublished).

${ }^{5}$ J. N. Bahcall, W. A. Fowler, I. Iben, Jr., and R. L. Sears, Astrophys. J. 137, 344 (1963). The central temperature of the sun for the theoretical model used in this paper (developed by Sears) is 16.2 million degrees. See also R. L. Sears, Mem. Soc. Roy. Sci. Liege $\underline{3}, 479$ (1960).

${ }^{6} \mathrm{P}$. Pochoda and $\mathrm{H}$. Reeves (to be published).

${ }^{7}$ R. L. Sears (to be published).

${ }^{8} \mathrm{~J}$. N. Bahcall (to be published). This reference will contain an extensive discussion of neutrino absorption cross sections that are relevant to the detection of solar neutrinos. A variety of experimental tests of the assumptions used to calculate the excited-state neutrino absorption cross sections for ${ }^{37} \mathrm{Cl}\left(\nu, e^{-}\right)^{37} \mathrm{Ar}$ will also be discussed.

${ }^{9}$ I am grateful to Professor B. R. Mottelson and Professor M. A. Preston for comments that sparked the investigation of excited-state transitions.

${ }^{10}$ The proton-proton $\left(q_{\nu} \max =0.42 \mathrm{MeV}\right)$ and ${ }^{7} \mathrm{Be}$ electron-capture $\left(q_{\nu}=0.86 \mathrm{MeV}\right)$ neutrinos do not have sufficient energy to induce transitions to excited states in ${ }^{37} \mathrm{Ar}$.

${ }^{11}$ The assumptions made in calculating the ${ }^{37} \mathrm{Cl}$ neutrino absorption cross sections could be directly checked by measuring the $f t$ values for the ${ }^{37} \mathrm{Ca} \rightarrow{ }^{37} \mathrm{~K}$ decays, one of whose branches is also superallowed. Two other experiments that would be useful in testing the assumptions made in the cross-section calcula- tions are (i) a measurement of the branching ratios in the ${ }^{37} \mathrm{~K} \rightarrow{ }^{37} \mathrm{Ar}$ decay, and (ii) a measurement with improved accuracy of the $f t$ values in the ${ }^{35} \mathrm{Ar} \rightarrow{ }^{35} \mathrm{Cl}$ decay. Predictions for the lifetimes and energies of all branches involved in the above decays are available upon request and will appear in reference 8.

${ }^{12}$ The possible importance of ${ }^{8} \mathrm{~B}$ solar neutrinos was first pointed out by W. A. Fowler, Astrophys. J. 127 , 551 (1958); A. G. W. Cameron, Ann. Rev. Nucl. Sci. $\underline{8}, 299$ (1958).

${ }^{13} \mathrm{See}$, for example, H. Greisen, Proceedings of International Conference for Instrumentation in High Energy Physics, Berkeley, California, September 1960 (Interscience Publishers, Inc., New York, 1961), p. 209 ; F. Reines, Ann. Rev. Nucl. Sci. 10, 1 (1960); B. Pontecorvo and Ya. Smorodinskii, Zh. Eksperim. i Teor. Fiz. 41, 239 (1961) [translation: Soviet Phys. - JETP $14, \overline{173}$ (1962)]. The preliminary experiment of Davis (reference 2) implies that the energy density of $1-\mathrm{MeV}$ cosmic neutrinos is less than $5 \mathrm{MeV} / \mathrm{cm}^{3}$; however, the galactic energy density of starlight is only about $1 \mathrm{eV} / \mathrm{cm}^{3}$. Thus the Davis experiment does not furnish a very stringent upper limit on the energy density of low-energy cosmic neutrinos.

${ }^{14} \mathrm{G}$. T. Zatsepin and V. A. Kuz'min, Zh. Eksperim. i Teor. Fiz. 41, 1818 (1961) [translation: Soviet Phys. -JETP 14, 1294 (1962)]; M. A. Markov and I. M. Zheleznykh, Nucl. Phys. 27, 385 (1961); T. D. Lee, H. Robinson, M. Schwartz, and R. Cool, Phys. Rev. 132, 1297 (1963).

${ }^{15}$ H. A. Bethe, Phys. Rev. $\underline{55}, 434$ (1939); H. Reeves, Phys. Rev. Letters $\underline{2}, 423(1959)$; S. Bashkin, R. W. Kavanagh, and P. D. Parker, Phys. Rev. Letters $\underline{3}$, 518 (1959). The possibility of terminating the protonproton chain through a particle-unstable but thermally populated ground state of ${ }^{4} \mathrm{Li}$ was apparently overlooked.

${ }^{16}$ Details of this calculation will appear in a paper by P. D. Parker, J. N. Bahcall, and W. A. Fowler (to be published). I am especially grateful to Dr. Parker for valuable collaboration on this point. Note that if ${ }^{4} \mathrm{Li}$ were particle stable, all proton-proton terminations in the sun would occur via ${ }^{3} \mathrm{He}(p, \gamma)^{4} \mathrm{Li}\left(\beta^{+} \nu\right)^{4} \mathrm{He}$ because of the relatively low Coulomb barrier for the ${ }^{3} \mathrm{He}(p, \gamma){ }^{4} \mathrm{Li}$ reaction and the high abundance of protons.

${ }^{17}$ This result implies that there are no $T=1$ alphaparticle bound states below $19 \mathrm{MeV}$. 